\title{
Molecular basis for cysteine oxidation by Plant Cysteine Oxidases from Arabidopsis thaliana
}

Qiong Guo ${ }^{1 \#}$, Zhenzhen Chen ${ }^{2 \#}$, Gao $\mathrm{Wu}^{1}$, Jie Wen ${ }^{2 *}$, Shanhui Liao ${ }^{1 *}$, Chao Xu${ }^{1 *}$

${ }^{1}$ Ministry of Education Key Laboratory for Membrane-less Organelles \& Cellular Dynamics, Hefei National Laboratory for Physical Sciences at the Microscale, School of Life Sciences, Division of Life Sciences and Medicine, University of Science and Technology of China, 230027 Hefei, P.R. China;

${ }^{2}$ The First Affiliated Hospital of University of Science and Technology of China (Anhui Provincial Hospital), 230000 Hefei, P.R. China

\#These authors contributed equally: Qiong Guo, Zhenzhen Chen

*Correspondence: Jie Wen (jiewen@ustc.edu.cn) or

Shanhui Liao (ajsod@mail.ustc.edu.cn) or Chao Xu (xuchaor@ustc.edu.cn)

Keywords: cysteine oxidation; crystal structure; Arg/N-degron; Cys-sulfinic acid; thiazolidine 


\begin{abstract}
Plant Cysteine Oxidases (PCOs) play important roles in controlling the stability of Group VII ethylene response factors (ERF-VIIs) via N-Arg/degron pathway through catalyzing the oxidation of their N-Cys for subsequent Arginyl-tRNA--protein transferase 1 (ATE1) mediated arginine installation. Here we presented structures of PCO2, PCO4, and PCO5 from Arabidopsis thaliana (AtPCOs) and examined their in vitro activity by MS. On the basis of Tris-bound AtPCO2, we modelled the Cys-bound AtPCO2 structure and identified key residues involved in N-Cys oxidation. Alanine substitution of potential N-Cys interaction residues impaired the activity of AtPCO5 remarkably. The structural research, complemented by mutagenesis and mass spectrometry experiments, not only uncovers the substrate recognition and catalytic mode by AtPCOs, but also sheds light on the future design of potent inhibitors for plant cysteine oxidases.
\end{abstract}




\section{Introduction}

In Animals and plants, different mechanisms are exploited to sense oxygen concentration and regulate gene expression in response to low oxygen stress (hypoxia)[1]. Animals utilizes hypoxia-inducible transcription factor (HIF) to increase the expression levels of growth factors [2], while Plant Cysteine Oxidases (PCOs) play a critical role in oxygen homeostasis by serving as oxygen-sensing proteins [3, 4]. PCOs were found to control the turnover of Group VII ethylene response factors (ERF-VIIs) starting with Met-Cys- $\left({ }^{1} \mathrm{MC}^{2}\right)$, via N-degron pathway[4-7]. Once the N-terminal Met (N-Met) of target protein is excised by Met amino peptidase[8], the exposed $\mathrm{N}$-terminal Cys (N-Cys) is subject to oxidization by PCOs, which covert the Cys to Cys-sulfinic acid $\left(\mathrm{CysO}_{2}\right)$ [7]. After the oxidation of N-Cys, Arginyl-tRNA--protein transferase 1 (ATE1) installs an arginine before $\mathrm{N}-\mathrm{CysO}_{2}$ to promote the proteasomal degradation through the Arg/N-degron pathway[4, 7].

The activity of PCOs were greatly compromised in the hypoxic condition, which promotes the expression of hypoxia-response genes by increasing the in vivo stability of the ERF-VIIs it mediated[5, 9]. Five ERF-VII family members were identified in Arabidopsis thaliana, including AtRAP2.2, AtRAP2.3, AtRAP2.12, AtHRE1, and AtHRE2 $[5,10]$. The turnovers of above response factors mediated by PCOs provide a new layer in understanding how gene expression during stress response is regulated in a oxygen-dependent manner.

PCOs from Arabidopsis thaliana (AtPCOs) has been found to catalyze the N-Cys oxidization of AtHRE1 ( $\left.{ }^{2} \mathrm{CGGAVIS}^{8}\right)$, AtHRE2 $\left({ }^{2} \mathrm{CGGAIIS}^{8}\right)$, and AtRAP2.2 $\left({ }^{2} \mathrm{CGGAIIS}^{8}\right)$, etc $[6,10]$. Despite their important biological functions in response to low-oxygen stress, how PCOs recognize the substrate and catalyze the subsequent NCys oxidation remain elusive because they display low sequence identity with the dioxygenases that have known structures. Here we solved several crystal structures of PCOs from Arabidopsis thaliana (AtPCOs), including those of AtPCO2, AtPCO4, and 
AtPCO5. Although the three PCOs belong to two subfamilies, they display conserved catalytic pocket, suggesting the conserved catalytic mechanism among PCOs. Specifically, the structure of Tris-bound AtPCO2 allows us to model an N-Cys in the catalytic pocket, which reveals the N-Cys specific recognition by PCOs. The PCOsmediated N-Cys oxidization was complemented by mass spectrometry (MS) experiments. Therefore, our structure research not only provides structural insights into the N-Cys catalytic mechanism by PCOs, but also sheds light on the future design of chemical inhibitors in interfering with the activities of PCOs.

\section{Results}

AtPCO2, AtPCO4, and AtPCO5 exhibit activities towards peptides derived from

\section{ERF-VII family members}

To study the activities of AtPCOs in vitro systematically, we cloned, expressed and purified the full-length or core fragment of several AtPCOs, including AtPCO248-276, AtPCO4 $_{1-241}$, and AtPCO51-242 (Fig. 1A). Then we synthesized two peptides derived from $A t \mathrm{HRE} 1$ and $A t \mathrm{RAP} 2.2, A t \mathrm{HRE} 1^{2-10}$ and $A t \mathrm{RAP} 2.2^{2-8}$, Respectively, and examine the activities of AtPCOs towards the two peptides by MS experiments.

Consistent with previous reports about the control experiments[5, 11], after long incubation without enzyme, the peptides either dimerized via N-Cys (Fig. 2A and 3A) or formed thiazolidine (Fig. 2B and 3B). The dimerized peptide and formation of thiazolidine were confirmed by the peaks correspond to the $\sim 2$-fold molecular weight and the $+12 \mathrm{Da}$, respectively. In contrast, both peptides were efficiently catalyzed by AtPCO248-276, At $\mathrm{PCO}_{1-241}$, and AtPCO51-242 in 30 minutes at $37^{\circ} \mathrm{C}$, which generated a peak of $+32 \mathrm{Da}$ (Fig. 2C-2E, and Fig. 3C-3E)

\section{Crystal structure of AtPCO51-242, AtPCO41-241, and AtPCO248-276}

To understand the substrate binding and catalytic mechanism of AtPCOs, we solved the crystal structure of selenomethionine (SeMet) AtPCO51-242 at a resolution of $2.50 \AA$ (Table 1). Most residues of $A t \mathrm{PCO}_{1-242}$ are visible except the residues at N-, C- 
terminus or some loop regions owning to internal flexibility (Fig. 1B). AtPCO5 adopts a jelly roll-like fold with a chamber constituted by two $\beta$-sheets, $\beta 14-\beta 1-\beta 2-\beta 9-\beta 4-\beta 7$ and $\beta 6-\beta 5-\beta 8-\beta 3$, with the former $\beta$-sheet packed against $\alpha 1-\alpha 3$ (Fig. 1B, 1C). There is a long bended insertion between $\beta 9$ and $\beta 14$ consisting of $\beta 10-\beta 13$, in which $\beta 10$ and $\beta 11$ form anti-parallel strands with $\beta 13$ and $\beta 12$, respectively. Before crystallization, we added excess ferrous ion in the buffer and the density map of a ferrous ion is found in the catalytic center, which is chelated to His98, His100, His164, and three water molecules. His 98 and His 100 reside in the loop between $\beta 3$ and $\beta 4$, while His 164 is localized in $\beta 8$ (Fig. 1).

Next we solved structures of $A t \mathrm{PCO} 4_{1-241}$ and $A t \mathrm{PCO} 2_{48-276}$, at resolutions of 2.44- and 1.56- $\AA$, respectively (Fig. 4A, 4B and Table 1). Both AtPCO4 and AtPCO2 adopt jelly roll-like fold (Fig. 4A, 4B). Superposition of three AtPCO structures show that their overall architecture are very similar, as evidenced by the root-mean-square deviations (RMSDs) in a range of 0.57-0.81 $\AA$ (Fig. 4C). Especially the three histidines that are chelated to the ferrous ion are conserved in three structures, as an indicative of the conserved ferrous ion chelation mode in AtPCOs (Fig. 4A, 4B). On the basis of sequence alignment, it is very likely that above (His) 3 chelation mode also applies for their human homolog, 2-aminoethanethiol dioxygenase (ADO) (Fig. 1A).

Intriguingly, in the structure of AtPCO2, we found that a Tris molecule, mimic of the $\mathrm{N}$-Cys, occupies the catalytic pocket. In the Tris-bound structure, besides the three histidines, the central ferrous ion is also chelated to the nitrogen and the two carboxyl groups of the Tris molecule to complete the six coordination (Fig. 4D). The nitrogen group of Tris is hydrogen bonded to the hydroxyl group of the AtPCO2 Tyr215. Tris also makes van del Walls interactions with Phe123, Ile131, Phe199, and Asp209 of AtPCO2 (Fig. 4D).

\section{Modeled structure of Cys-bound AtPCO2}

Given the similarity between the Tris and the apo cysteine, we modeled the Cys-bound 
structure of AtPCO2 on the basis of the Tris-bound structure and compared its Cys recognition mode with that of recently solved Cys-bound human CDO (PDB ID: 6N42)[12]. Despite the similar architecture and Ferrous ion chelation mode, the jellyroll fold of CDO deviates remarkably from that of AtPCO2 (Fig. 5A). At the catalytic center, the modelled Cys is chelated to the $\mathrm{Fe}^{2+}$ via its main chain amino and side chain thiol groups, similar to that observed in the structure of Cys-bound CDO (Fig. 5B, 5C). Despite the similarity, their Cys recognition and catalytic modes are different in several aspects. Firstly, Cys93 and Tyr157 of CDO forms the cysteinyltyrosine bridge to increase the catalytic efficiency (Fig. 5B) $[12,13]$, whereas the crosslinked moiety is not found in any of AtPCO structures (Fig. 5C). Secondly, the nitrogen group of Cys in the Cys-CDO complex is free, whereas the nitrogen group of Cys is hydrogen bonded to the side chain of Tyr215 in the modeled AtPCO2 complex (Fig. 5B, 5C). Thirdly, in both structures, the Cys interaction residues in two structures are distinct (Fig. 5B, 5C). In the CDO structure, the Cys makes van der Waals interactions with the side chains of Leu75, Ser83, Val142, and His155 (Fig. 5B). In the modelled complex structure of AtPCO2, the Cys makes van der Waals interactions with the side chains of Phe123, Ile131, and Phe199 of AtPCO2 and its side chain makes one hydrogen bond with the side chain carboxyl group of Asp209, positioning the Cys in a favorable conformation for oxidation (Fig. 5C).

The Cys interaction residues of AtPCO2 are absolutely conserved in AtPCO4 and AtPCO5 (Fig. 1A), suggesting a common Cys recognition and catalytic mode shared by AtPCOs. The counterparts of AtPCO2 Asp209 and Tyr215 in AtPCO5 are Asp176 and Tyr182, respectively (Fig. 1A). We then generated several single mutants for AtPCO51-242, including H164A, D176A, Y182F and C190A, and examined their activities towards the peptide RAP2.2 $2^{2-8}$ (CGGAIIS). MS data show that while H164A abolished the activity by disrupting the ferrous chelation, both D176A and Y182F demonstrated compromised activities towards the AtHRE1 $1^{2-10}$ and AtRAP2.2-8 peptides than wild type AtPCO5. In contrast, C190A exhibit comparable activity towards the substrate peptide as the wild type (Supplementary Fig. S1). Collectively, 
the mutagenesis experiments and MS data further confirmed the key roles of the Cys binding residues in cysteine oxidation.

\section{Discussion}

Previous work on human $\mathrm{CDO}$ reveal that an cysteinyltyrosine bridge is formed to lower the oxidation potential of tyrosine for efficient catalysis[12, 13]. Whether cysteinyltyrosine bridge is also formed in human ADO and PCOs remains unknown because of the absence of structure evidence. By analyzing the structure of AtPCOs, we proposed that although Cys 190 of AtPCO2 is spatially adjacent to Tyr215 and Tyr225 and the three residues are absolutely conserved in the structures of AtPCO4 and AtPCO5 (Fig. 6), it could not form the cysteinyltyrosine bridge with either of them. Firstly, the the cysteinyltyrosine bridge of CDO (Cys93-Tyr157) does not have a spatial counterpart in AtPCO2. The Cys190 of AtPCO2 is not the counterpart of CDO Cys92 in CDO (Fig. 5B, 5C). Secondly, the aromatic ring of AtPCO2 Tyr225 is far from the thiol group of Cys223, implied that they are not likely to form crosslinked moiety. (Fig. 6). Thirdly, as for the Tyr215 of AtPCO2, it is not likely to cross link with Cys223, either, because its aromatic ring of Tyr215 is parallel to the side chain plain of Cys223 and it would be energetically unfavorable for the Try2 15 to rotate its aromatic right at least $~ 90$ degree to form cysteinyltyrosine bridge. Also the main chain carbonyl group of Pro214 and the main chain amino group of Ser216 are hydrogen bonded to the side chain of Arg221 and the main chain carbonyl group of Arg221, respectively (Fig. 6), which does not allow the Tyr215 main chain to endure remarkable change. Above mentioned spatial pattern of the Cys223, Tyr215, and Tyr225 of AtPCO2 are also conserved in AtPCO4 and AtPCO5 (Fig. 6), suggesting that AtPCOs probably exploit a different mechanism rather than cysteinyltyrosine bridge to catalyze cysteine oxidation. We further mutating the Cys190 of AtPCO4, the counterpart of the AtPCO2 Cys223, to an Ala, and found that C190A does not impair the AtPCO4 activity towards RAP2.2 $2-8$ (Supplementary Fig. S1).

During the preparation of the manuscript, the structures of AtPCO4 and AtPCO5 from 
Arabidopsis thaliana were reported[14]. In that study, the presented structures, as well as the conclusions drawn on the basis of the structures, are similar to ours. In near future, the substrate- or inhibitor- bound structures of AtPCOs would further uncover the catalytic mechanism of N-Cys oxidation.

\section{Conclusion}

Plant Cysteine Oxidases from Arabidopsis thaliana have been reported to play an important role in controlling the turnover of response factors via $\mathrm{Arg} / \mathrm{N}$-degron pathway. Our presented structures of AtPCO2, AtPCO4, and AtPCO5 unraveled the architecture of PCOs, and the structure of ligand-bound AtPCO2 revealed the potential substrate binding and oxidation residues in the catalytic pocket. Besides, sequence alignment indicates that human ADO might utilize a mechanism to catalyze the oxidation of $\mathrm{N}$ Cys similar to those of AtPCOs. Overall our research not only provides insight into the $\mathrm{N}$-Cys oxidation mechanism by PCOs, but also sheds light on the design of inhibitors for PCOs.

Acknowledgements: We thank the staff at beam line BL18U1 of the Shanghai Synchrotron Radiation Facility for providing technical support and assistance in data collection and analysis. This work was supported by the "Strategic Priority Research Program" of the Chinese Academy of Sciences (Grant No. XDB19000000) and National Natural Science Foundation of China Grants (31770806, 31500601). C. X. is also supported by the Major/Innovative Program of the Development Foundation of the Hefei Center for Physical Science and Technology (2018CXFX007) and the "Thousand Young Talent program".

Declarations of interest: The authors declare that they have no conflict of interest Author contributions: Q.G. and C.X. designed and conceived the study. Q.G., Z.C., S.L. purified, crystallized the protein, solved the structure, and analyzed the data. Q.G. and G.W. performed MS experiments. C.X. wrote the paper with the help from all authors. J.W., S.L., and C.X. supervised the experiments. 


\section{Materials and Methods}

\section{Cloning, mutation, protein expression and purification}

Genes encoding full-length AtPCO2, AtPCO4, and AtPCO5 were synthesized by Sangon Biotech (Shanghai). AtPCO248-276, AtPCO41-241, and AtPCO51-242 was amplified by polymerase chain reactions (PCR), and cloned into pET28-MHL (Genbank accession number: EF456735). The plasmids were then transformed into E. coli BL21 (DE3) and the recombinant proteins were overexpressed at $16{ }^{\circ} \mathrm{C}$ for $20 \mathrm{~h}$ in the presence of $0.2 \mathrm{mM}$ isopropyl b-D-1-thiogalactopyranoside (IPTG).

Cells were harvested at $3600 \times \mathrm{g}, 4{ }^{\circ} \mathrm{C}$ for $15 \mathrm{~min}$, and then were resuspended using a buffer containing $20 \mathrm{mM}$ Tris- $\mathrm{HCl}(\mathrm{pH} 7.5), 400 \mathrm{mM} \mathrm{NaCl}$ (suspension buffer) and were lysed by sonication. Lysates were centrifuged at $18000 \times \mathrm{g}, 4^{\circ} \mathrm{C}$ for $30 \mathrm{~min}$ and supernatants were collected. Recombinant proteins were purified with a fast flow NiNTA column (GE Healthcare) and eluted by $20 \mathrm{mM}$ Tris- $\mathrm{HCl}$ (pH 7.5), $400 \mathrm{mM} \mathrm{NaCl}$, $500 \mathrm{mM}$ imidazole. Gel filtration and ion-exchange were employed for further purification. Gel filtration experiments were performed on a HiLoad ${ }^{\mathrm{TM}}$ 16/ 600 superdex ${ }^{\mathrm{TM}} 75 \mathrm{pg}$ column (GE healthcare) with suspension buffer, the fractions containing target recombinant proteins were collected and dialyzed to ion exchange buffer A (20 mM Tris- $\mathrm{HCl} \mathrm{pH} 7.5,50 \mathrm{mM} \mathrm{NaCl})$. Ion exchange experiments were performed on a Hitrap ${ }^{\mathrm{TM}}$ Q HP (1 mL) column (GE healthcare) with ion exchange buffer $\mathrm{A}$ and ion exchange buffer $\mathrm{B}(20 \mathrm{mM}$ Tris- $\mathrm{HCl} \mathrm{pH} 7.5,1 \mathrm{M} \mathrm{NaCl})$, fraction corresponding to target proteins were collected and concentrated to $20-40 \mathrm{mg} / \mathrm{mL}$ and stored at $-80^{\circ} \mathrm{C}$ before further use. Seleno-Methionine (SeMet)-labeled AtPCO5 1-242 was purified in the same way, except that cells were cultured in M9 medium supplied with 50mg/L Seleno-Methionine. The mutants were constructed by conventional PCR using a MutanBEST kit (TaKaRa) and further verified by DNA sequencing. The mutants were expressed and purified in the same way as the wild type proteins.

\section{Crystallization, data collection and structure determination}


Before crystallization, recombinant proteins were pre-incubated with $\mathrm{Fe}^{2+}$ at a molar ratio of $1: 3$ at $4{ }^{\circ} \mathrm{C}$ for $30 \mathrm{~min}$. For crystallization of AtPCOs, $1 \mu$ l protein was mixed with $1 \mu$ crystallization buffer using the sitting drop vapor diffusion method at $18{ }^{\circ} \mathrm{C}$. SeMet-labeled AtPCO51-242 was crystallized in a buffer containing 0.1 M MES monohydrate, $\mathrm{pH}$ 6.5, $12 \%(\mathrm{w} / \mathrm{v})$ PEG20000. AtPCO4 ${ }_{1-241}$ was crystallized in a buffer containing 0.1 M Sodium citrate tribasic dehydrate, $\mathrm{pH}$ 5.0, 0.2 M Ammonium acetate, and 20\% (w/v) PEG 3350. AtPCO248-276 was crystallized in a buffer containing $0.1 \mathrm{M}$ Tris hydrochloride, $\mathrm{pH} 8.5,0.2 \mathrm{M}$ sodium acetate, and 30\% w/v polyethylene glycol 4000. Before flash-freezing crystals in liquid nitrogen, all crystals were soaked in a cryo-protectant consisting of $90 \%$ reservoir solution plus $10 \%$ glycerol.

The diffraction data were collected on beam line BL18U1 at the Shanghai Synchrotron Facility (SSRF). Data sets were collected and processed using the HKL3000 program[15]. The initial model of SeMet AtPCO5 $5_{1-242}$ was solved by CRANK2[16], built manually by COOT[17] and refined by Phenix[18]. The structures of AtPCO248-276 and AtPCO41-241 were solved by molecular replacement by using the structure of SeMet AtPCO5 $1-242$ as the search model. The structures were built manually by Coot[17] and were further refined by Phenix [18].

The atomic coordinates and structure factors of AtPCO2, AtPCO4, and AtPCO5 have been deposited in the Protein Data Bank with PDB ID codes 7CHJ, 7CHI, and 7CXZ.

\section{Mass spectrometry experiments}

Reversed-phase microcapillary/tandem mass spectrometry (LC/MS/MS) was performed using an Easy-nLC nanoflow HPLC (Proxeon Biosciences) with a selfpacked $75 \mathrm{~mm} 15 \mathrm{~cm} \mathrm{C18}$ column connected to a QE-Plus (Thermo Scientific) in datadependent acquisition and positive ion mode at $300 \mathrm{~nL} / \mathrm{min}$. Passing MS/MS spectra were manually inspected to ensure that all b-and y-fragment ions aligned with the assigned sequence and modification sites. A $25 \mu \mathrm{l}$ reaction mixture contained $1 \mu \mathrm{M}$ AtPCO2/4/5 (final concentration) and $50 \mu \mathrm{M}$ peptide (final concentration) in a buffer containing $20 \mathrm{mM}$ Tris- $\mathrm{HCl}$ (pH 8.0), $20 \mathrm{mM} \mathrm{NaCl}, 20 \mu \mathrm{M} \mathrm{FeSO}_{4}, 5 \mathrm{mM}$ TCEP, and 
$1 \mathrm{mM}$ Ascorbic acid. The reaction was incubated at $37^{\circ} \mathrm{C}$ for $30 \mathrm{~min}$ at atmospheric oxygen before being quenched (at $70^{\circ} \mathrm{C}$ for $10-15$ mins). Then, the tubes were centrifuged at $15000 \times \mathrm{g}$ for $10 \mathrm{~min}$. Supernatants were analyzed by LC-MS/MS and Proteomics Browser software, with the relative abundances of substrate and product reflecting the cysteine oxidation activities of proteins.

\section{References}

1 Majmundar, A. J., Wong, W. J. and Simon, M. C. (2010) Hypoxia-inducible factors and the response to hypoxic stress. Mol Cell. 40, 294-309

2 Kaelin, W. G., Jr. and Ratcliffe, P. J. (2008) Oxygen sensing by metazoans: the central role of the HIF hydroxylase pathway. Mol Cell. 30, 393-402

3 Masson, N., Keeley, T. P., Giuntoli, B., White, M. D., Puerta, M. L., Perata, P., Hopkinson, R. J., Flashman, E., Licausi, F. and Ratcliffe, P. J. (2019) Conserved N-terminal cysteine dioxygenases transduce responses to hypoxia in animals and plants. Science. 365, 65-69

4 Dissmeyer, N. (2019) Conditional Protein Function via N-Degron Pathway-Mediated Proteostasis in Stress Physiology. Annu Rev Plant Biol. 70, 83-117

5 White, M. D., Klecker, M., Hopkinson, R. J., Weits, D. A., Mueller, C., Naumann, C., O'Neill, R., Wickens, J., Yang, J., Brooks-Bartlett, J. C., Garman, E. F., Grossmann, T. N., Dissmeyer, N. and Flashman, E. (2017) Plant cysteine oxidases are dioxygenases that directly enable arginyl transferase-catalysed arginylation of $\mathrm{N}$-end rule targets. Nat Commun. 8, 14690

6 White, M. D., Kamps, J., East, S., Taylor Kearney, L. J. and Flashman, E. (2018) The plant cysteine oxidases from Arabidopsis thaliana are kinetically tailored to act as oxygen sensors. J Biol Chem. 293, 11786-11795

7 Varshavsky, A. (2019) N-degron and C-degron pathways of protein degradation. Proc Natl Acad Sci U S A. 116, 358-366

8 Ye, Q. Z., Xie, S. X., Ma, Z. Q., Huang, M. and Hanzlik, R. P. (2006) Structural basis of catalysis by monometalated methionine aminopeptidase. Proc Natl Acad Sci U S A. 103, 9470-9475

9 Licausi, F., Kosmacz, M., Weits, D. A., Giuntoli, B., Giorgi, F. M., Voesenek, L. A., Perata, P. and van Dongen, J. T. (2011) Oxygen sensing in plants is mediated by an $\mathrm{N}$-end rule pathway for protein destabilization. Nature. 479, 419-422

10 Weits, D. A., Giuntoli, B., Kosmacz, M., Parlanti, S., Hubberten, H. M., Riegler, H., Hoefgen, R., Perata, P., van Dongen, J. T. and Licausi, F. (2014) Plant cysteine oxidases control the oxygen-dependent branch of the N-end-rule pathway. Nat Commun. 5, 3425

11 Kallen, R. G. (1971) The mechanism of reactions involving Schiff base intermediates. Thiazolidine formation from L-cysteine and formaldehyde. J Am Chem Soc. 93, 6236-6248

12 Ye, S., Wu, X., Wei, L., Tang, D., Sun, P., Bartlam, M. and Rao, Z. (2007) An insight into the mechanism of human cysteine dioxygenase. Key roles of the thioether-bonded tyrosine-cysteine cofactor. J Biol Chem. 282, 3391-3402

13 Joseph, C. A. and Maroney, M. J. (2007) Cysteine dioxygenase: structure and mechanism. Chem Commun (Camb), 3338-3349

14 White, M. D., Dalle Carbonare, L., Lavilla Puerta, M., lacopino, S., Edwards, M., Dunne, K., Pires, E., Levy, C., McDonough, M. A., Licausi, F. and Flashman, E. (2020) Structures of Arabidopsis thaliana 
oxygen-sensing plant cysteine oxidases 4 and 5 enable targeted manipulation of their activity. Proc Natl Acad Sci U S A

15 Minor, W., Cymborowski, M., Otwinowski, Z. and Chruszcz, M. (2006) HKL-3000: the integration of data reduction and structure solution--from diffraction images to an initial model in minutes. Acta Crystallogr D Biol Crystallogr. 62, 859-866

16 Pannu, N. S., Waterreus, W. J., Skubak, P., Sikharulidze, I., Abrahams, J. P. and de Graaff, R. A. (2011) Recent advances in the CRANK software suite for experimental phasing. Acta Crystallogr D Biol Crystallogr. 67, 331-337

17 Emsley, P. and Cowtan, K. (2004) Coot: model-building tools for molecular graphics. Acta Crystallogr D Biol Crystallogr. 60, 2126-2132

18 Adams, P. D., Grosse-Kunstleve, R. W., Hung, L. W., loerger, T. R., McCoy, A. J., Moriarty, N. W., Read, R. J., Sacchettini, J. C., Sauter, N. K. and Terwilliger, T. C. (2002) PHENIX: building new software for automated crystallographic structure determination. Acta Crystallogr D Biol Crystallogr. 58, 1948-1954 
Table 1 Data collection and refinement statistics

\begin{tabular}{|c|c|c|c|}
\hline & SeMet $A t$ PCO5 & AtPCO4 & AtPCO2 \\
\hline \multirow[t]{2}{*}{ Data collection } & Beamline SSRF & Beamline SSRF & Beamline SSRF \\
\hline & BL18U1 & BL18U1 & BL18U1 \\
\hline Radiation wavelength $(\AA)$ & 0.9792 & 0.9792 & 0.9792 \\
\hline Space group & $\mathrm{P} 4_{1} 2_{1} 2$ & $\mathrm{P} 12{ }_{1} 1$ & C 121 \\
\hline \multicolumn{4}{|l|}{ Cell dimensions } \\
\hline $\mathrm{a}, \mathrm{b}, \mathrm{c}(\AA)$ & $\begin{array}{c}67.72,67.72 \\
128.32\end{array}$ & $\begin{array}{c}56.62,51.70 \\
82.06\end{array}$ & $\begin{array}{c}91.07,46.82 \\
56.13\end{array}$ \\
\hline$\alpha, \beta, \gamma\left(^{\circ}\right)$ & $90,90,90$ & $90,108.06,90$ & $90,100.94,90$ \\
\hline Resolution $(\AA)$ & $\begin{array}{c}50.00-2.50(2.59- \\
2.50)\end{array}$ & $\begin{array}{c}50.00-2.45(2.54- \\
2.44)\end{array}$ & $\begin{array}{c}50.00- \\
1.56(1.59-1.56)\end{array}$ \\
\hline $\mathrm{R}_{\text {merge }}$ & $0.135(1.625)$ & $0.119(0.344)$ & $0.053(0.194)$ \\
\hline $\mathrm{I} / \sigma \mathrm{I}$ & $32.6(4.0)$ & $14.2(4.0)$ & $31.9(8.6)$ \\
\hline $\mathrm{CC}_{1 / 2}$ & $0.985(0.894)$ & $0.993(0.941)$ & $0.999(0.968)$ \\
\hline Completeness (\%) & $100.0(100.0)$ & $99.2(94.5)$ & $100.0(100.0)$ \\
\hline Redundancy & $24.4(21.5)$ & $6.4(5.4)$ & $6.7(6.1)$ \\
\hline \multicolumn{4}{|l|}{ Refinement } \\
\hline Resolution $(\AA)$ & $24.72-2.50$ & $31.14-2.44$ & $25.14-1.56$ \\
\hline Number of unique reflections & 19650 & 26952 & 33140 \\
\hline $\mathrm{R}_{\text {work }} / \mathrm{R}_{\text {free }}$ & $0.180 / 0.226$ & $0.205 / 0.266$ & $0.157 / 0.185$ \\
\hline \multicolumn{4}{|l|}{$\begin{array}{l}\text { Number of atoms/B-factor } \\
\left(\AA^{2}\right)\end{array}$} \\
\hline Proteins & $1698 / 43.3$ & $3525 / 40.3$ & $1923 / 15.9$ \\
\hline Metal ions & $1 / 17.3$ & $2 / 31.1$ & $2 / 10.8$ \\
\hline Others & - & $13 / 56.1$ & $8 / 9.7$ \\
\hline Water & $62 / 42.4$ & $94 / 33.5$ & $263 / 25.7$ \\
\hline $\operatorname{RMSD}$ bonds $(\AA) / \operatorname{angles}\left({ }^{\circ}\right)$ & $0.009 / 1.02$ & $0.003 / 0.56$ & $0.009 / 1.00$ \\
\hline \multicolumn{4}{|l|}{ Ramachandran Plot } \\
\hline favored/allowed/outliers (\%) & $95.8 / 4.2 / 0.0$ & $97.3 / 2.7 / 0.0$ & $97.5 / 2.5 / 0.0$ \\
\hline
\end{tabular}

Values in parentheses are for highest-resolution shell. 


\section{Figure legends}

Figure 1. Structure of AtPCO5. (A) Sequence alignment of AtPCOs and human ADO ( $h \mathrm{ADO})$. Secondary structures of AtPCO5 were labelled in blue at the top of aligned sequences. Three $\mathrm{Fe}^{2+}$ chelating histidines (His98, His100, and His164), and residues potentially involved in N-Cys recognition are labeled. (B) Overall structure of AtPCO51-242. AtPCO5 is colored in blue, with three $\mathrm{Fe}^{2+}$ chelating histidines (His98, His100, and His 164) shown in sticks. $\mathrm{Fe}^{2+}$ and three water molecules are shown in orange and red, respectively. (C) Topology diagram of AtPCO5, with secondary structures colored in blue.

Figure 2. Mass spectra data of peptide $A t \mathrm{HRE} 1^{2-10}$ (CGGAVISDY) catalyzed by AtPCOs. (A)-(B) Spectra of peptide without enzyme as the controls. Spectra the peptide CGGAIIS oxidized by (C) AtPCO51-242, (D) AtPCO41-241, and (E) AtPCO248-276.

Figure 3. Mass spectra data of peptide AtRAP2.2-8 (CGGAIIS) catalyzed by AtPCOs. (A)-(B) Spectra of peptide without enzyme as the controls. Spectra the peptide CGGAIIS oxidized by (C) AtPCO51-242, (D) AtPCO41-241, and (E) AtPCO248-276.

Figure 4. Structures of $A t \mathrm{PCO} 4_{1-241}$, and $A t \mathrm{PCO} 2_{48-276}$. (A) Overall structure of AtPCO4 ${ }_{1-241}$. The structure of AtPCO4 is shown in red cartoon, with three $\mathrm{Fe}^{2+}$ chelating histidines (His98, His100, and His164) shown in sticks. $\mathrm{Fe}^{2+}$ and three water molecules are shown in orange and red, respectively. (B) Overall structure of Tris-bound AtPCO248-276. The structure of $A t \mathrm{PCO} 2$ is colored in cyan cartoon, with three $\mathrm{Fe}^{2+}$ chelating histidines (His134, His136, and His197) shown in sticks. $\mathrm{Fe}^{2+}$ and Tris are shown in orange and yellow, respectively. (C) Superposition of the structures of AtPCO2 (cyan), AtPCO4 (red), and AtPCO5 (blue). The structures are shown in ribbon, with $\mathrm{Fe}^{2+}$ chelation residues shown in sticks. (D) Detailed interactions between Tris and AtPCO2. Tris is shown in yellow sticks. $\mathrm{Fe}^{2+}$ chelating residues and the Tris-binding residues are shown in cyan sticks. Intermolecular hydrogen bond is indicated by black dashes. 
Figure 5. Comparison of the catalytic mechanism of AtPCOs with that of human CDO. (A) Superposition of the structure of $h \mathrm{CDO}$ (red) with that of AtPCO2 (cyan). The proteins are shown in ribbon, with $\mathrm{Fe}^{2+}$ chelating residues shown in sticks. (B) The catalytic center of Cys-bound $h \mathrm{CDO}$, with the Cys and Cys binding residues shown sticks. (C) The catalytic center of Cys-bound AtPCO2, with the Cys and Cys binding residues shown sticks.

Figure 6. Spatial pattern of Cys223, Tyr215, and Tyr225 of AtPCO2. Pro214 and Ser216 form main chain hydrogen bonds with $\mathrm{Arg} 221 . \mathrm{Fe}^{2+}$ chelating residues, $\mathrm{Arg} 221$, Cys223, Pro214-Ser216, Tyr225, as well as their counterparts in AtPCO4 and AtPCO5, are shown in sticks. 
VSEEALDKVRNVIEKIKPSDVGLEQEAQLVRNWPGPGN . . ERNGNHHSLPAIKYIQIHE

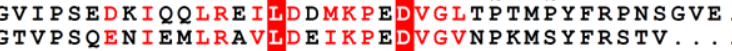

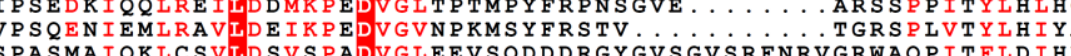

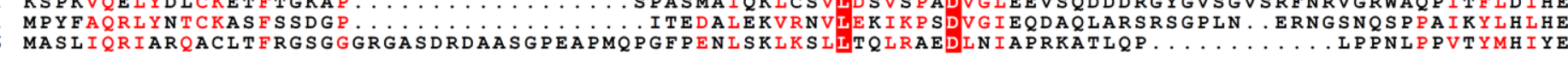

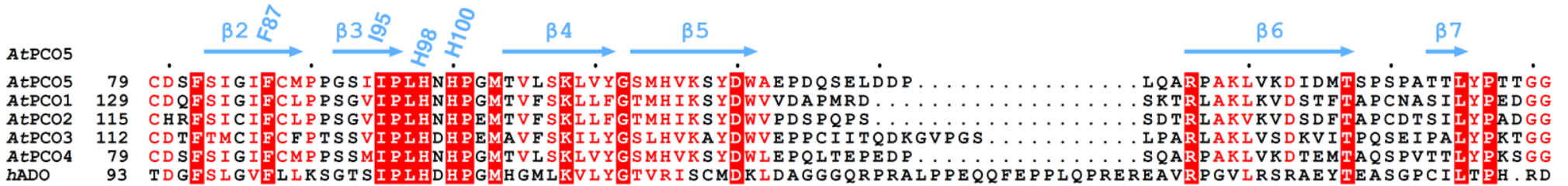

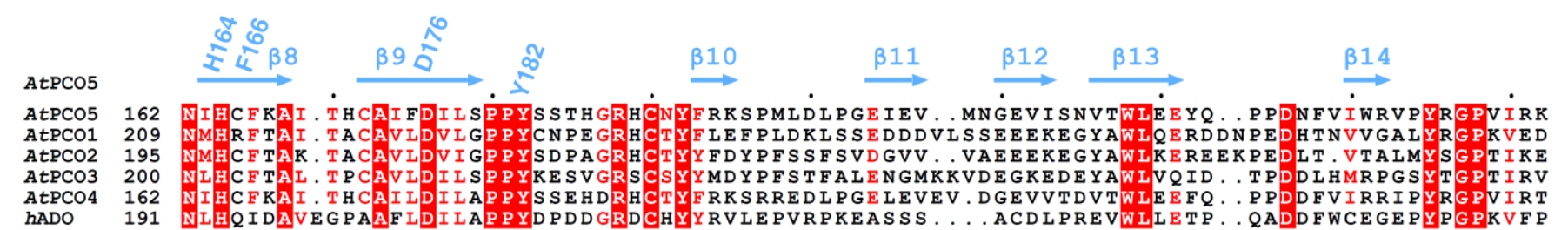

B

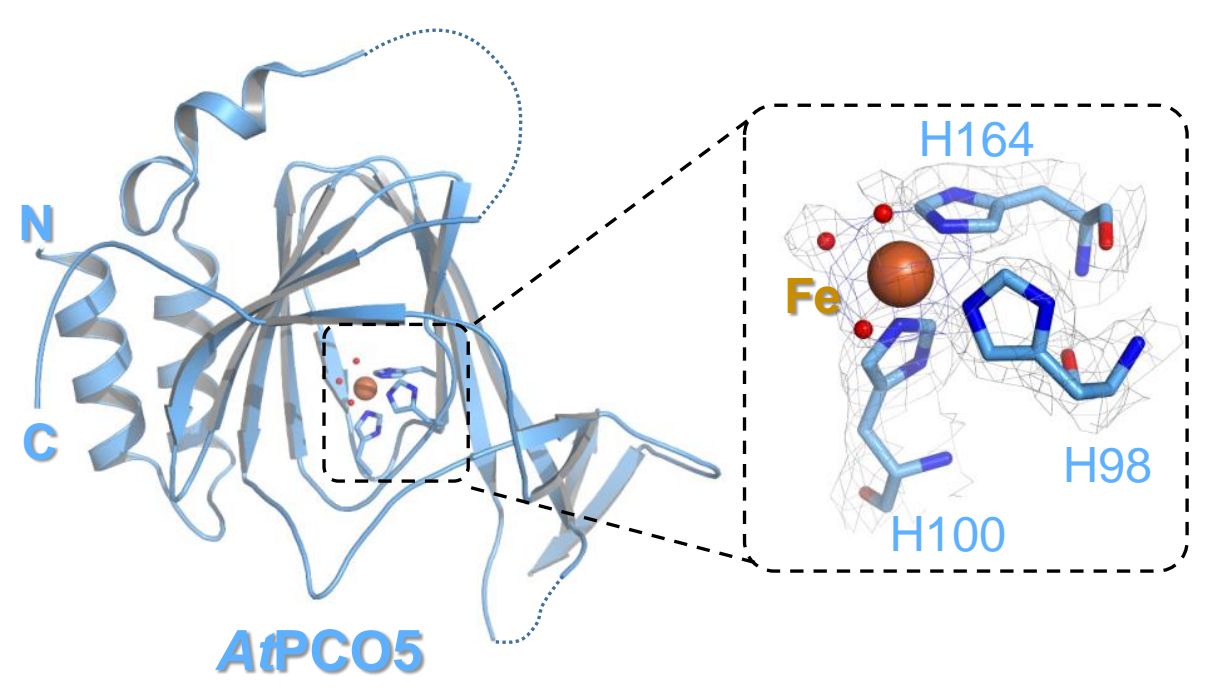

C

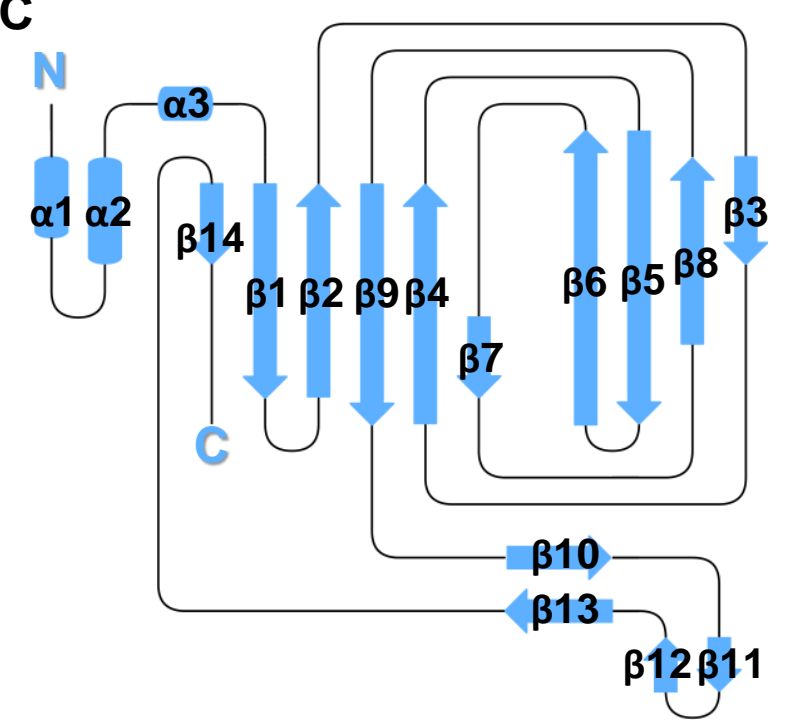



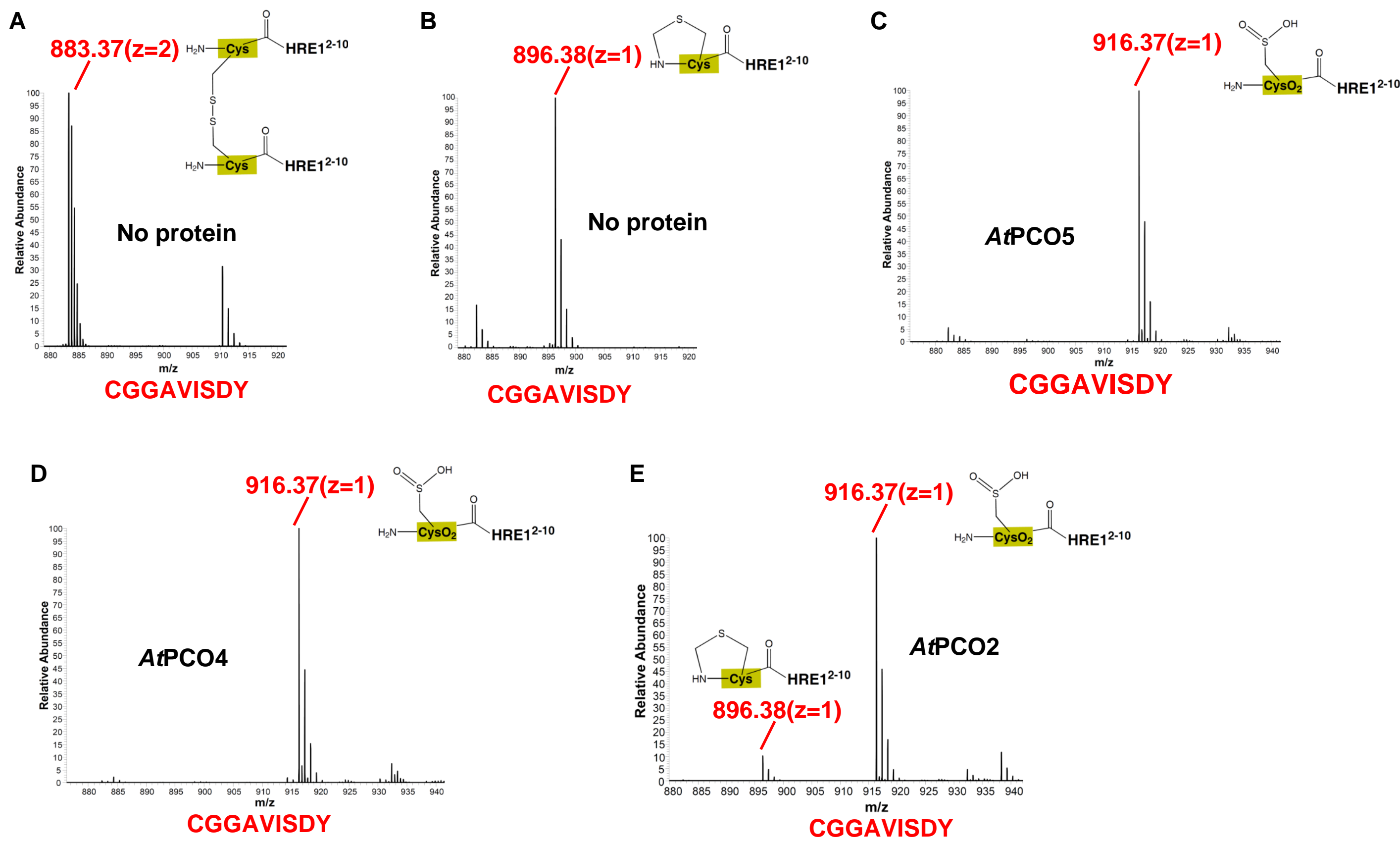

\section{Figure 2}


A

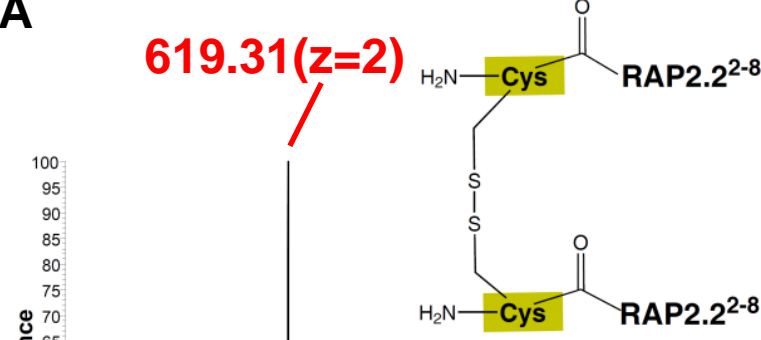

No protein

D

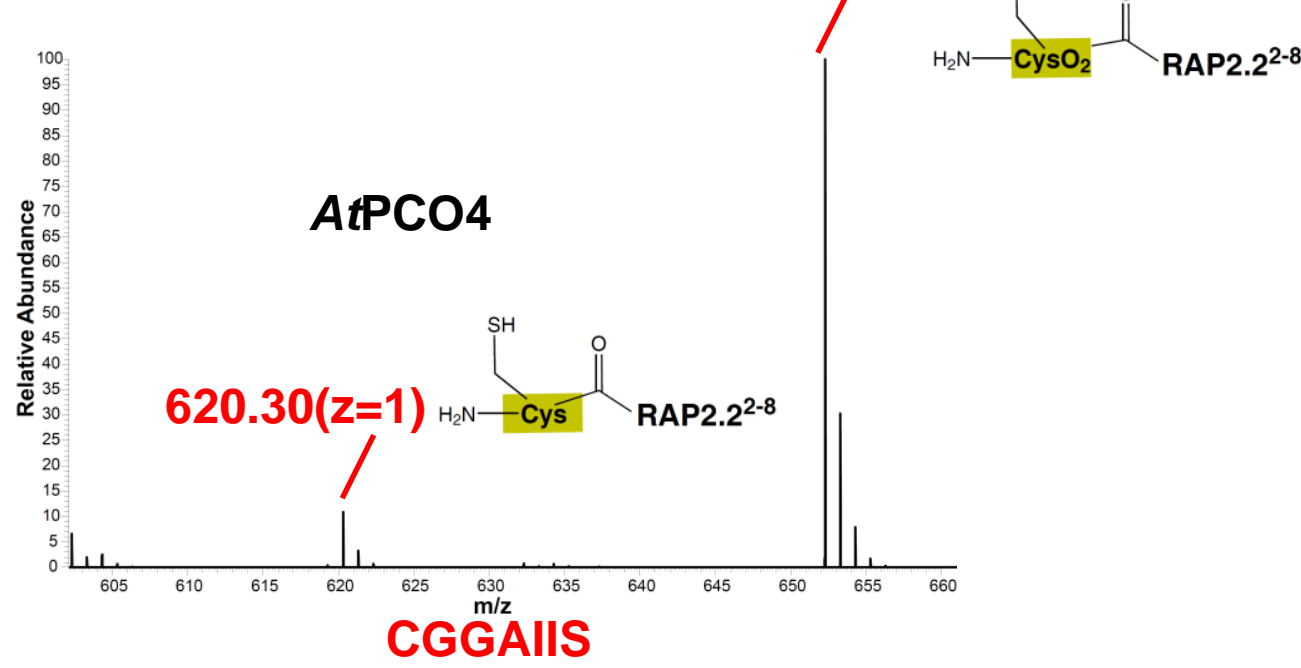

B
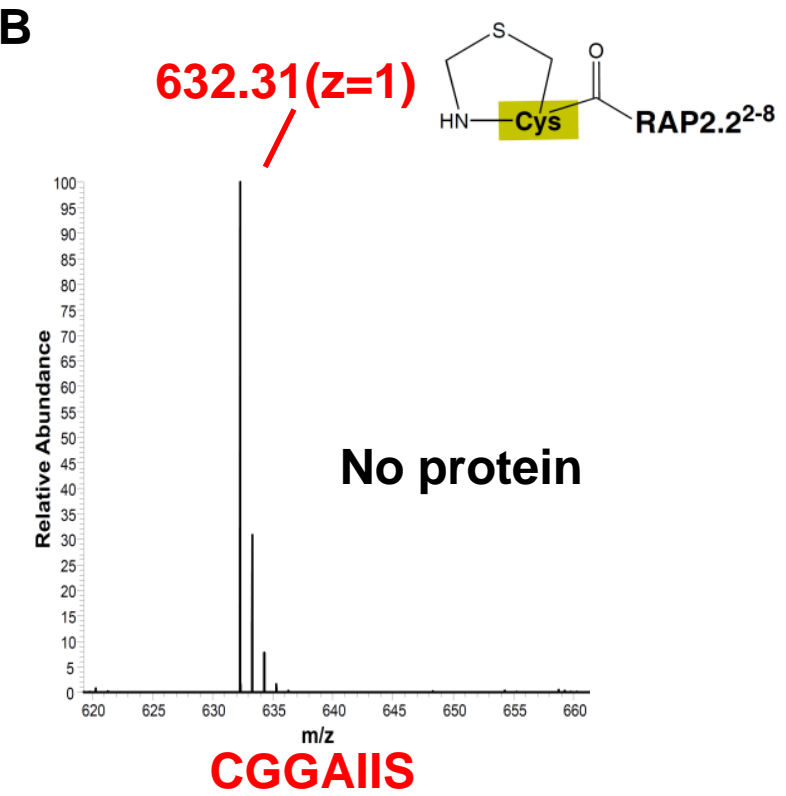

C

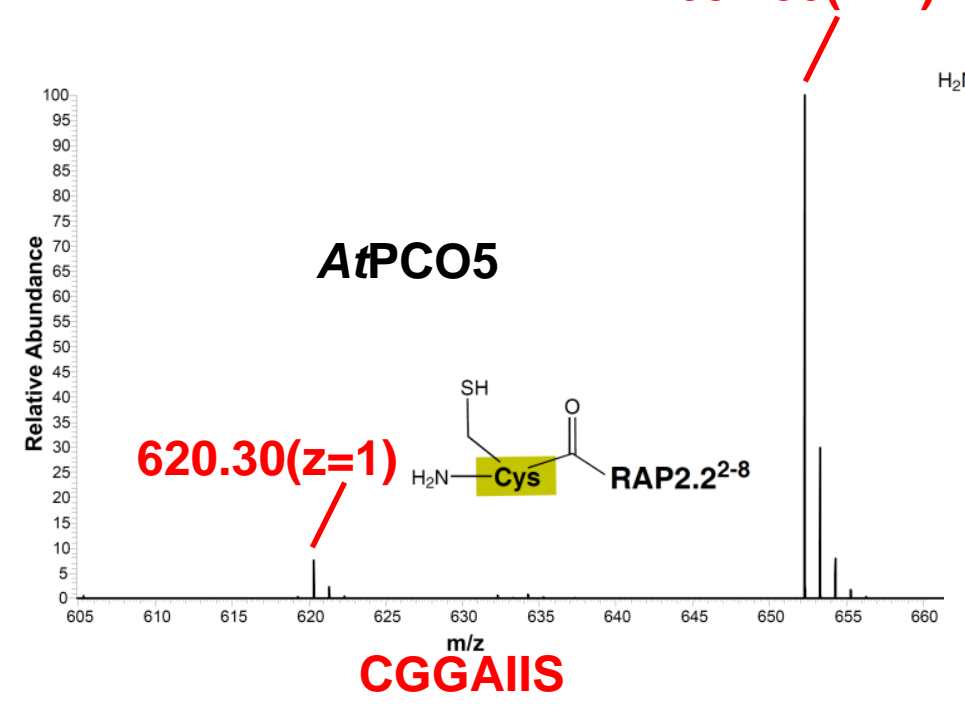

E

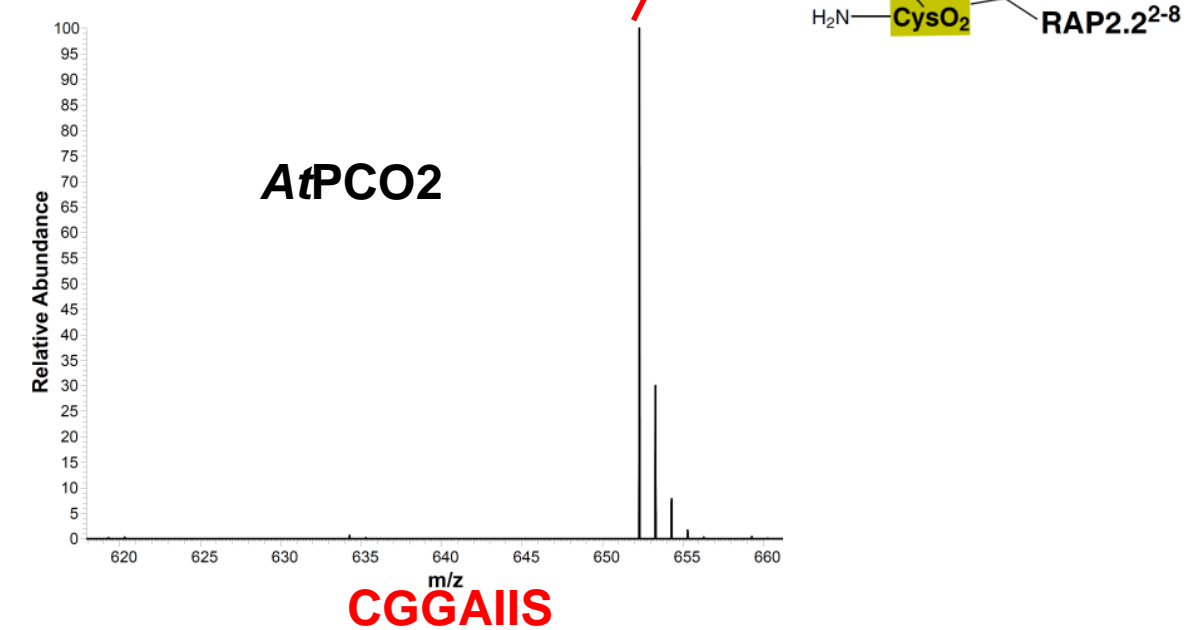

\section{Figure 3}


A

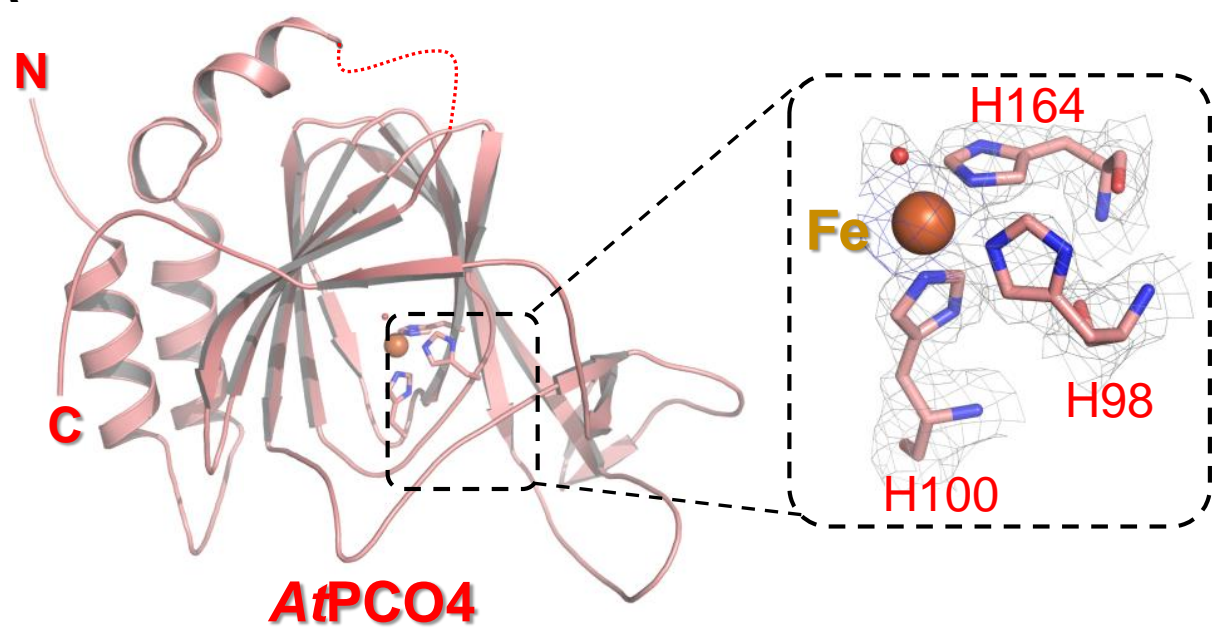

C

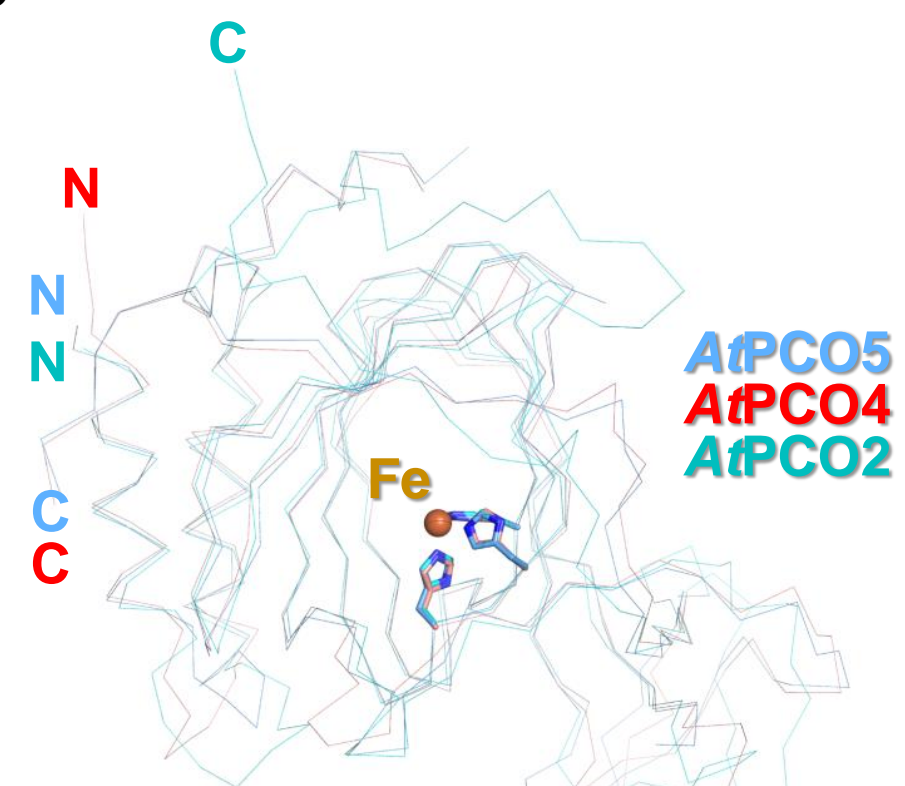

D
B
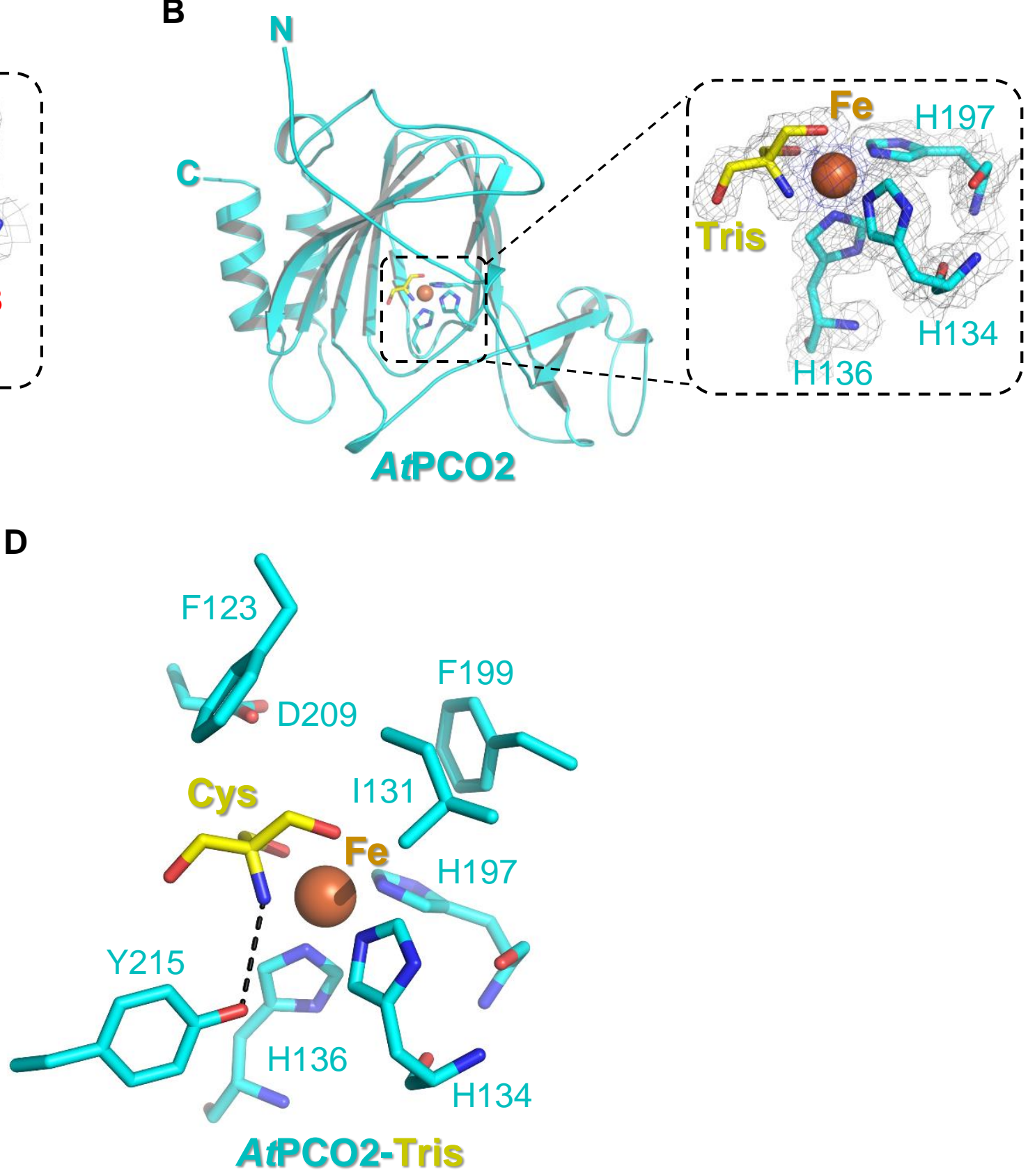
A

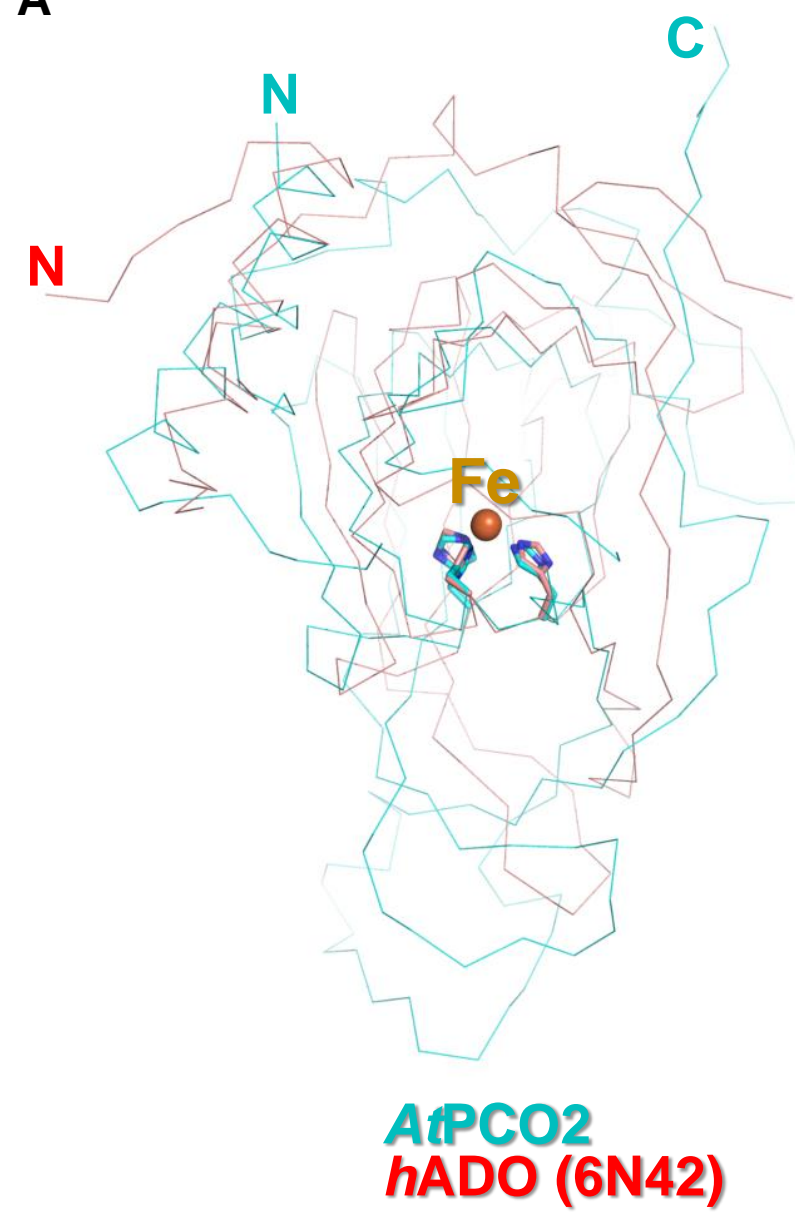

B

C

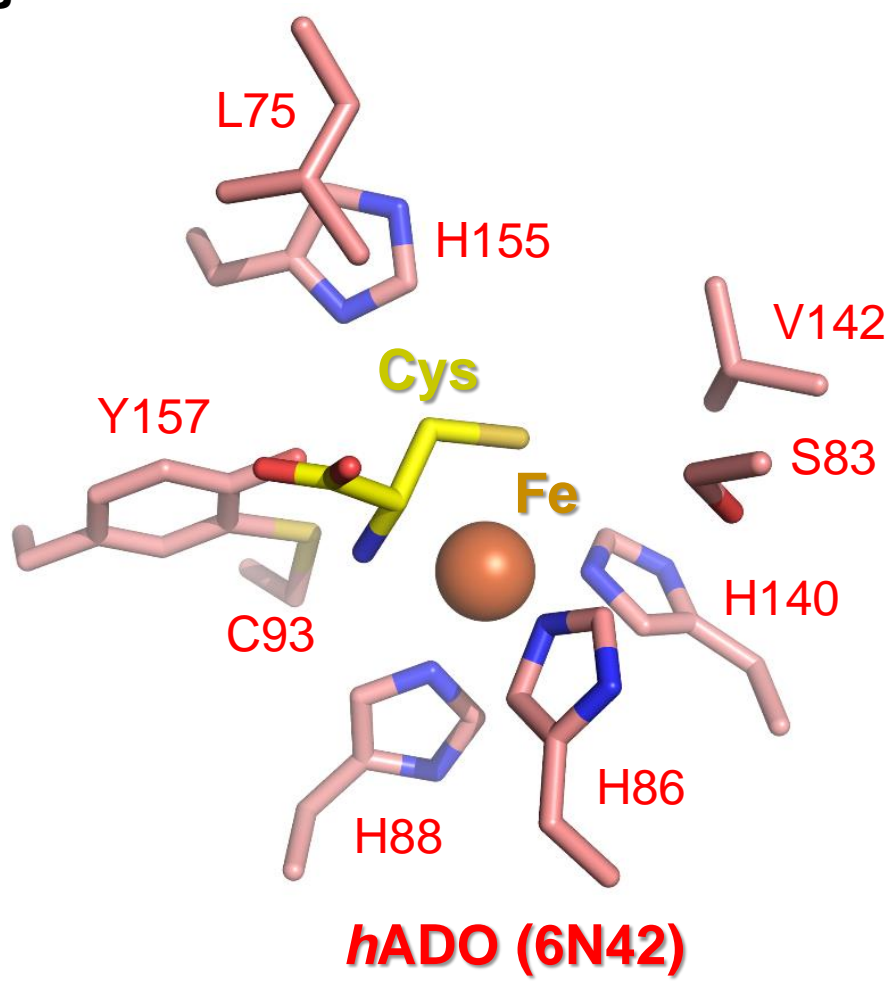

C

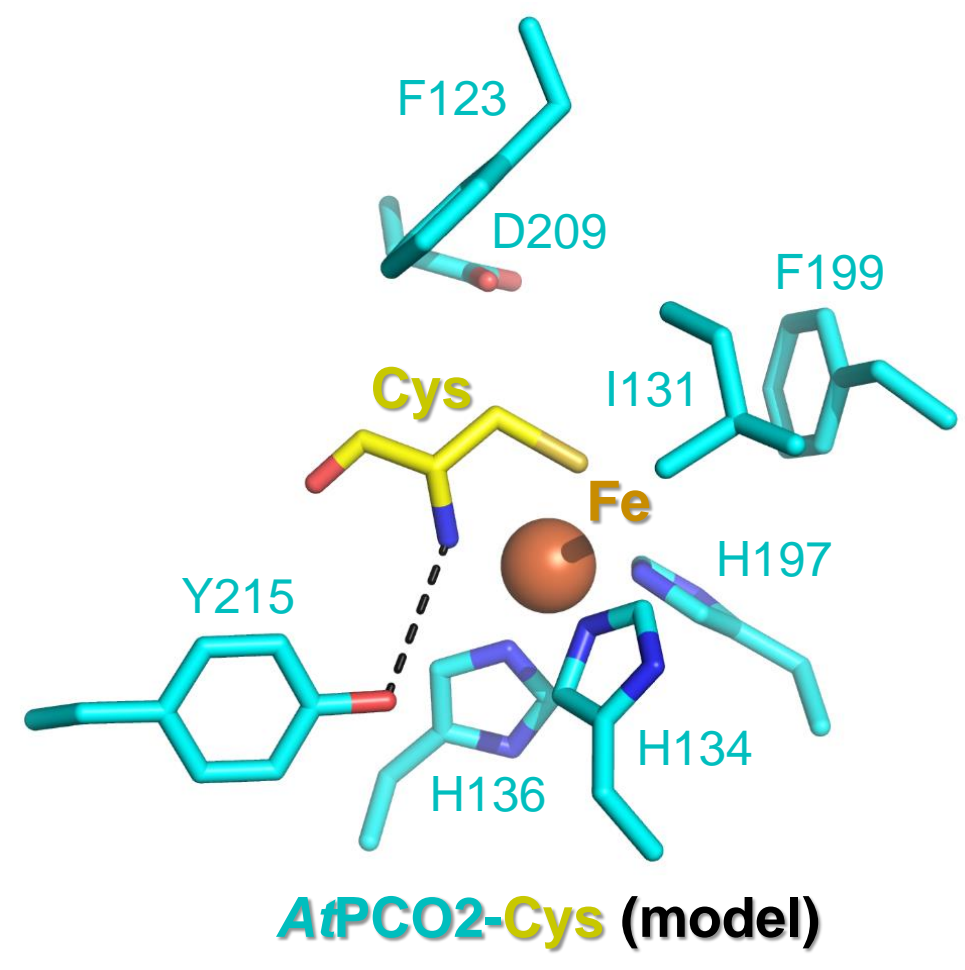




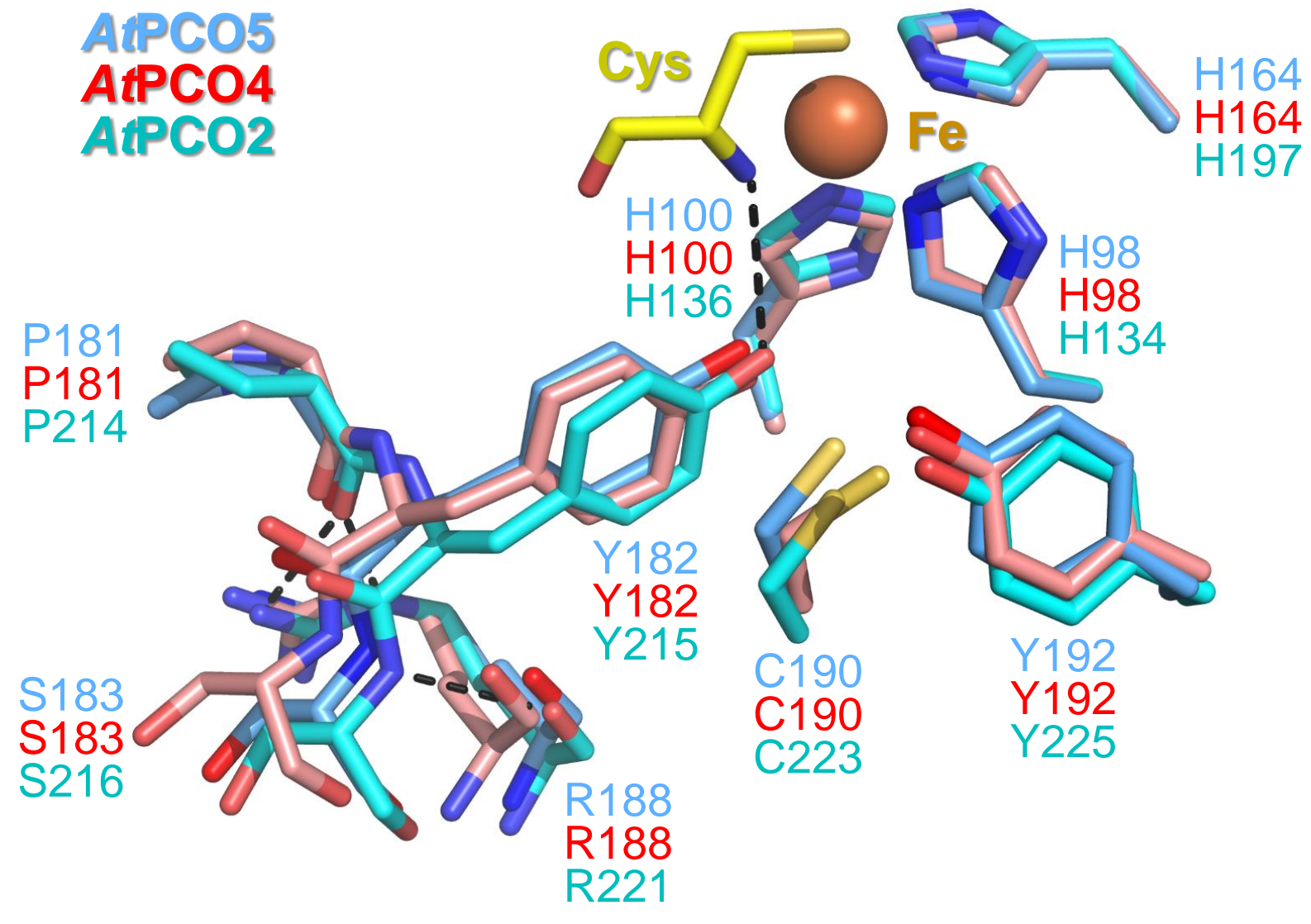

\section{Figure 6}



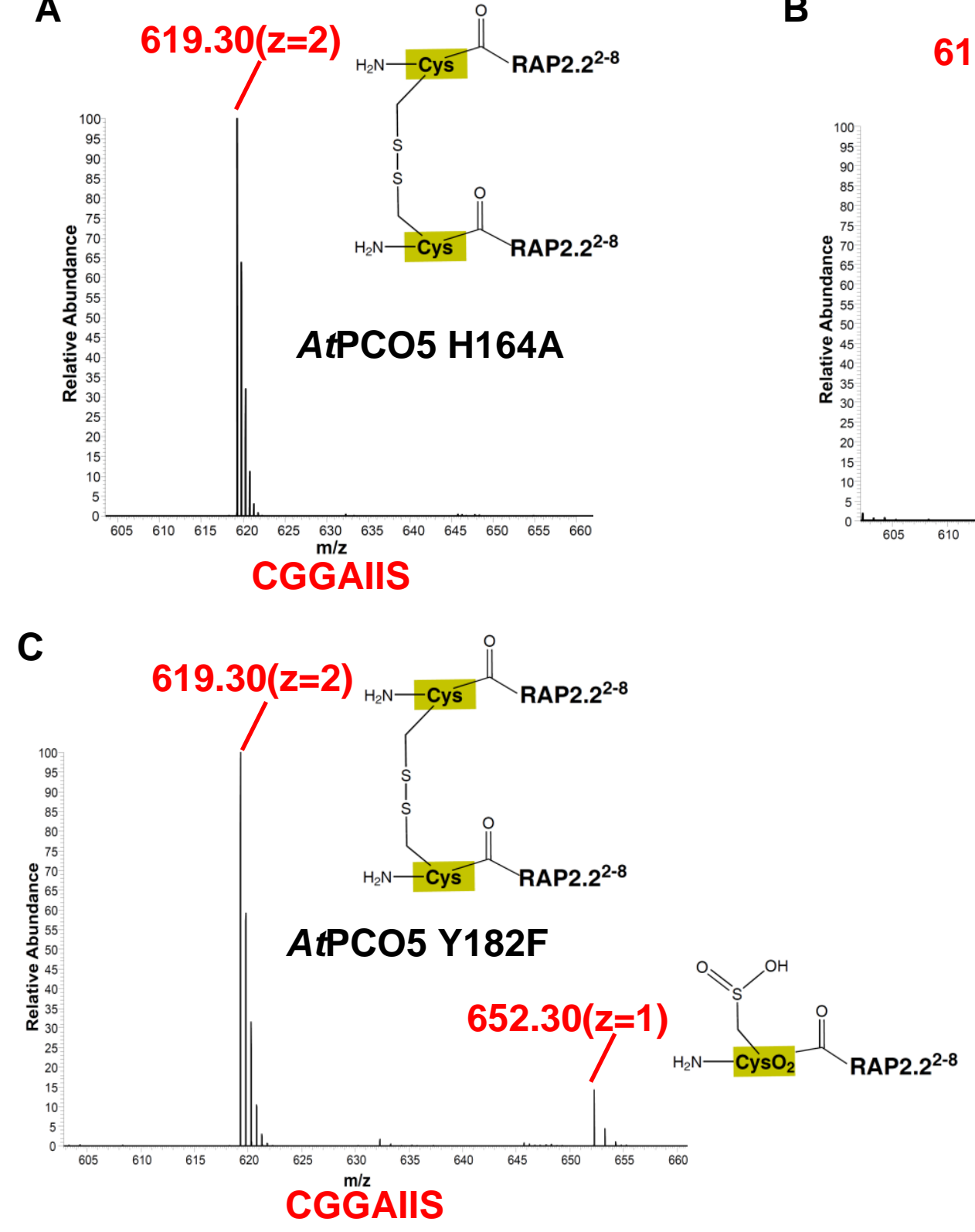

Supplementary Fig. S1. Mass spectra data of peptide AtRAP2.22-8 (CGGAIIS) catalyzed by the variants of AtPCO5. Spectra of peptide catalyzed by (A) AtPCO5 ${ }_{1-242} \mathrm{H} 164 \mathrm{~A}$, (B) AtPCO5 ${ }_{1-242} \mathrm{D} 176 \mathrm{~A}$, (C) AtPCO5 ${ }_{1-242} \mathrm{Y} 182 \mathrm{~F}$, and (D) AtPCO5 ${ }_{1-242} \mathrm{C} 190 \mathrm{~A}$. 